\title{
Strehl Ratio and Myopia in Chinese Adolescents: The Tuyou County Pediatric Eye (TYPE) Study
}

\author{
Xiaoyan Bian ${ }^{1, *}$ \\ Yuxia Guo ${ }^{2, *}$ \\ Shixuan Guo ${ }^{3, *}$ \\ Song Zhang ${ }^{4, *}$ \\ Guisen Zhang $\mathbb{( D}^{3}$ \\ Lei Liu (iD) ${ }^{4}$ \\ Yajun Yang $\mathbb{D}^{5}$ \\ Jun Liu ${ }^{6}$
}

'Department of Ocular Surface, Baotou Chaoju Eye Hospital, Baotou, 014060, People's Republic of China; ${ }^{2}$ Department of Cataract Surgery, Ninghai Eye Hospital, Ningbo, 3156I5, People's Republic of China; ${ }^{3}$ Department of Retina, Hohhot Chaoju Eye Hospital, Hohhot, 010050, People's Republic of China; ${ }^{4}$ Department of Ophthalmology, The First Affiliated Hospital of China Medical University, Shenyang, II000I, People's Republic of China; ${ }^{5}$ Department of Cataract, Baotou Chaoju Eye Hospital, Baotou, 0I4060, People's Republic of China; ${ }^{6}$ Department of Ophthalmology, Changzhi People's Hospital, Changzhi, 046000, People's Republic of China

*These authors contributed equally to this work

Correspondence: Yajun Yang

Department of Cataract, Baotou Chaoju Eye Hospital, Baotou, 014060, People's Republic of China

Email yangyajun@chaojueye.com

Jun Liu

Department of Ophthalmology, Changzhi People's Hospital, Changzhi, 046000,

People's Republic of China

Email yuanliu97@sina.com
Aim: To investigate the relationships between property of the visual quality, Strehl ratio (SR) and the degree of myopia.

Methods: A total of 444 anatomically normal eyes of 222 adolescents were enrolled in the TYPE study. Based on spherical equivalent (SE), subjects were divided into four groups: emmetropia/control (SE: +0.75 to $-0.75 \mathrm{D}$ ), low myopia (SE: -0.75 to $-3.00 \mathrm{D}$ ), moderate myopia (SE: -3.00 to $-5.00 \mathrm{D}$ ), high myopia (SE: $<-5.00 \mathrm{D})$. Axial length (AL) was measured. SR was attained with an OPD-III SCAN and calculated under a $3 \mathrm{~mm}$ pupil diameter.

Results: The overall SR (mean \pm SD) was $0.40 \pm 0.08$. Among all included eyes, the SR in eyes with the emmetropia, low myopia, moderate myopia and high myopia was $0.80 \pm 0.11$, $0.31 \pm 0.04,0.21 \pm 0.11$, and $0.11 \pm 0.02$, respectively. Furthermore, the $\mathrm{K} 2$ in eyes with the emmetropia, low myopia, moderate myopia and high myopia was $43.83 \pm 1.50,43.96 \pm 1.37$, $43.4 \pm 5.52$, and $45.16 \pm 1.43$, respectively. Significant differences were detected within the four groups in terms of SR and K2 $(P<0.001)$. Multiple regression analysis confirmed that AL negatively affected SR independently $(P<0.001)$.

Conclusion: Our findings provide a useful basis for the conclusion that myopia affects visual quality SR in Chinese adolescents. Besides, when performing visual quality SR, axial length must be taken into consideration, as it will influence SR.

Keywords: myopia, visual quality, SR, axial length

\section{Introduction}

In recent years, myopia has gained increasing interest, particularly because of its increase in prevalence across populations worldwide. ${ }^{1}$ Myopia is not a simple refractive error, but threatens people's visual acuity and visual quality., ${ }^{2,3}$

LASIK surgery can correct myopia, and the changes in optical quality after LASIK surgery depended on the patient's preoperative optical quality. Herein, previous studies have shown that myopia seriously affects the visual quality in adults and the higher the degree of myopia, the lower the visual quality. ${ }^{4,5}$ Strehl ratio (SR) is one of the visual image quality metrics and has been shown to be predictive of subjective best focus. ${ }^{6,7}$ Besides, the researches on metrics of visual quality including SR are mainly focused on the changes of visual quality in myopia patients before and after refractive surgery intervention. ${ }^{8,9}$ However, there is a lack of population-based studies on the visual quality of children with myopia, this study adopts the population-based cross-sectional design to analyze the visual quality of adolescents with myopia. 


\section{Methods}

The school-based, cross-sectional Tuyou Pediatric Eye (TYPE) study was carried out from September 1 to December 15, 2019, in the Tuyou County of Baotou in the Inner Mongolia Autonomous Region, China. The detailed information of the TYPE study was published in elsewhere. ${ }^{10}$ This study was a section of the TYPE study and aimed to investigate the relationship between myopia and visual quality (Strehl ratio) in adolescents aged 11-18 years. Briefly, this study adopts the multi-stage cluster sampling strategy and selects two middle schools (junior high school and senior high school). First of all, in view of the serious imbalance in China's development, Tuyou County in Baotou City in northern China represents an administrative division and is elected from the Inner Mongolia Autonomous Region of China. Second, select a high school and a junior high school from the suburban of Tuyou County as the representatives of education level. Finally, two classes are randomly selected from each grade of each school. All 230 students from these classes were included in the survey and eligible for all examinations; 8 students did not acquire full data, and the remaining 222 students (96.5\%, 444 eyes) completed the questionnaire and all eye examinations without cycloplegia. We included subjects with spherical equivalent (SE) less than +0.75 diopter (D), a best-corrected visual acuity (BCVA) greater than or equal to 1.0 units in the logarithm of minimal angle of resolution $(\log \mathrm{MAR})$ scale, transparent cornea, and those without any evidence of strabismus or intraocular disease as determined by an ophthalmologist and stopped wearing contact lenses for more than 2 weeks. Exclusion criteria were listed as follows: 1) suffering with systemic immune system diseases and other eye diseases that affect vision; 2) with a history of eye surgery or trauma. Subgroups were categorized according to their SE values, with 217 eyes in emmetropia group $(+0.75 \mathrm{D}<\mathrm{SE}<-0.75 \mathrm{D})$ and 134 eyes in the low myopia group $(-0.75 \mathrm{D}<\mathrm{SE}<-3.0 \mathrm{D}) ; 55$ eyes in the moderate myopia group $(-3.0 \mathrm{D}<\mathrm{SE}<-5.0 \mathrm{D})$, and 38 eyes in the high myopia group ( $\mathrm{SE} \geq-5.0 \mathrm{D}$ ). In this cross-sectional study, 444 eyes of 222 adolescents aged $13.52 \pm 0.64$ years (mean \pm standard deviation (SD), range: $11-<18$ years) were enrolled in the current study. The study complied with the Declaration of Helsinki and was approved by the institutional review board of Baotou Chaoju Eye Hospital (No. CHAOJU-BT-2020003), and all participants or their parents gave written informed consent. This study followed the Strengthening the Reporting of Observational Studies in Epidemiology (STROBE) guidelines (https://www.bmj.com/content/335/7624/806).

\section{Demographic Characteristics}

Demographic information, including age, sex, family socioeconomic status, and lifestyle, such as sleep duration per day, were collected via questionnaires. Sleep duration per day was ascertained using the following question, "During your longest or nocturnal sleep period, what time do you normally go to bed and wake up?" (Time was recorded in minute format).

\section{Body Check}

Each participant underwent a comprehensive body examination including weight, height, systolic blood pressure (SBP), diastolic blood pressure (DBP) and heart ratio. Body mass index (BMI) was calculated by dividing body weight in kilograms by the square of the height in meters.

\section{Ocular Examination}

Further, a comprehensive ophthalmologic examination was conducted including best-corrected visual acuity (BCVA) testing, ocular biometry including (axial length, corneal keratometry such as $\mathrm{K} 1, \mathrm{~K} 2$ and $\mathrm{Km}$ ) with the IOL Master 500 (Zeiss Meditec, Inc., Dublin, CA), fundus examination using nonmydriatic cameras TRC-NW8 (Topcon Corporation, Tokyo, Japan). SE was calculated as the sphere plus half the cylindrical power. Intraocular pressure (IOP) was measured with a non-contact tonometer (Nidek NT-510, Nidek, Aichi, Japan).

Optical visual quality was performed with the OPD IIISCAN (NIDEK, Japan). The machine can obtain rootmean-square (RMS) values at a pupil diameter of $4.0 \mathrm{~mm}$. The SR, calculated by means of the optical transfer function method, captures the effectiveness of the retinal point spread function (PSF) at stimulating the neural portion of the visual system.

\section{Definition}

The SR is the ratio of central intensity of the point image between the measured eye and the ideal eye. A value of 1 indicates a perfect system influenced only by diffraction. ${ }^{11}$ SR indicates the ratio of the light intensity of the Gaussian image point between defective and perfect refractive media, which is 0.15 in healthy adults; the higher the value, the better is the visual quality. ${ }^{8}$

\section{Statistical Analysis}

Statistical analysis was performed using the SPSS software, version 23.0 (IBM Corp, USA). Data obtained from 
each group were compared using 1-way analysis of variance (ANOVA). Univariate regression analysis was used to determine relationships between systematic variables (eg, age, sex, SBP, DBP, and BMI) and SR. Multivariate regression analysis coefficients $(\beta)$ were used to assess age, sex, and interaction in models for SR. P values were 2 sided, and $\mathrm{P}$ value less than 0.05 was considered statistically significant.

\section{Results}

There were significant differences in BMI $(P=0.036)$, SBP $(P=0.041)$ AL $(P<0.001)$ and SR $(P<0.001)$ according to whether myopia or not. However, there were no significant differences between participants with or without myopia in IOP, $\mathrm{K} 1, \mathrm{~K} 2$, and $\mathrm{Km}$, respectively (all $P>0.05$, Table 1).

Significant differences were detected within the degree of myopia groups in terms of heart ratio $(P=0.005)$, AL $(P<0.001)$, SR $(P<0.001)$ and $\mathrm{K} 2(P=0.004)$. Besides, SR showed a significant decrease in the severity of myopia $(P<0001)$. However, there were no significant differences between the myopic groups in IOP, $\mathrm{K} 1$, and $\mathrm{Km}$, respectively (all $P>0.05$, Table 2 ).

Associations between demographic, systemic, and ocular variables with SR metrics by univariate and multiple regression analyses are shown in Table 3 . In the multiple regression model adjusted with age and gender, AL was independently associated with SR $(\beta=-0.415, P<0.001)$

\section{Discussion}

In the current study, we investigated the relationship between SR and degree of myopia in adolescents with the following demographic or systematic characteristics: age, sex, sleep duration per day, AL, intraocular pressure, SBP, DBP, height, weight, and BMI. According to the analysis of the research results, there was a significant difference for SR in four groups according to their spherical equivalent refractive error. Moreover, SR showed a weak but significant negative correlation with AL.

There is no population-based evidence on the correlation between SR and myopia. Previously, Kamiya et al conducted a study on healthy volunteers aged 20-69 years and found that objective visual quality parameters, such as the modulation transfer function cutoff frequency $\left(\mathrm{MTF}_{\text {cutoff }}\right)$ and SR decreased significantly with age, while the objective scatter index (OSI) increased significantly according to the optical quality analysis system II (OQAS II). ${ }^{12}$ However, all participants of the current study were healthy young people with an age range of 11-18 years. The permeability of the refractive medium was normal and did not affect the quality of retinal imaging and intraocular scattering. Therefore, the visual quality of healthy young participants with myopia is not affected by age.

In addition, this study also found that after adjusting for confounders, SR was negatively correlated with AL $(P<0.001)$. This suggests that the visual quality of adolescents

Table I Adolescent Characteristics by Whether There is Myopia or Not

\begin{tabular}{|c|c|c|c|c|}
\hline Variables & Overall $(n=444)$ & Myopic Eyes $(n=227)$ & Normal Eyes $(n=2 \mid 7)$ & $\boldsymbol{P}$ \\
\hline Age (years) & $13.52 \pm 0.64$ & $13.56 \pm 0.70$ & $13.47 \pm 0.57$ & 0.138 \\
\hline Gender (boy, \%) & $208(46.84)$ & $97(46.63)$ & III(53.37) & 0.076 \\
\hline Height $(\mathrm{cm})$ & $163.25 \pm 34.66$ & $165.68 \pm 47.85$ & $|60.7| \pm 7.43$ & 0.131 \\
\hline Weight (kg) & $53.65 \pm 12.32$ & $52.69 \pm 12.30$ & $54.65 \pm 12.29$ & 0.093 \\
\hline BMI $\left(\mathrm{kg} / \mathrm{m}^{2}\right)$ & $20.61 \pm 4.19$ & $20.21 \pm 4.38$ & $21.04 \pm 3.95$ & 0.036 \\
\hline $\mathrm{SBP}(\mathrm{mmHg})$ & $111.89 \pm 13.00$ & $113.18 \pm 13.64$ & $110.65 \pm 12.17$ & 0.041 \\
\hline $\mathrm{DBP}(\mathrm{mmHg})$ & $66.23 \pm 9.318$ & $66.18 \pm 9.12$ & $66.28 \pm 9.53$ & 0.914 \\
\hline Heart ratio & $83.74 \pm 12.28$ & $83.53 \pm 12.23$ & $83.96 \pm 12.35$ & 0.716 \\
\hline Sleeping time per day (min) & $514.12 \pm 63.03$ & $511.04 \pm 69.76$ & $517.35 \pm 55.10$ & 0.072 \\
\hline $\mathrm{IOP}(\mathrm{mmHg})$ & $|6.79 \pm 8.0|$ & $16.84 \pm 10.89$ & $16.73 \pm 2.74$ & 0.887 \\
\hline Axial length (mm) & $23.82 \pm 1.72$ & $24.32 \pm 2.16$ & $23.29 \pm 0.80$ & $<0.001$ \\
\hline SR & $0.40 \pm 0.08$ & $0.11 \pm 0.03$ & $0.80 \pm 0.11$ & $<0.001$ \\
\hline KI & $44.29 \pm 26.38$ & $44.69 \pm 25.50$ & $43.88 \pm 27.33$ & 0.119 \\
\hline K2 & $43.93 \pm 2.39$ & $44.02 \pm 3.01$ & $43.83 \pm 1.50$ & 0.059 \\
\hline $\mathrm{Km}$ & $44.11 \pm 13.29$ & $44.35 \pm 12.85$ & $43.86 \pm 13.76$ & 0.693 \\
\hline
\end{tabular}

Note: Data are presented as mean \pm SD.

Abbreviations: BMI, body mass index; DBP, diastolic blood pressure; IOP, intraocular pressure; SBP, systolic blood pressure; SR, Strehl ratio; KI, horizontal axes; K2, vertical axes; $\mathrm{Km}$, average corneal curvature. 
Table 2 Adolescent Characteristics by Severity of Myopia

\begin{tabular}{|c|c|c|c|c|c|c|}
\hline Variables & $\begin{array}{l}\text { Overall } \\
(n=444)\end{array}$ & $\begin{array}{c}\text { Emmetropia } \\
(n=217) \\
(-0.75 \mathrm{D} \\
<\text { SE < +0.75 D) }\end{array}$ & $\begin{array}{c}\text { Low Myopia } \\
\quad(n=134) \\
(-0.75 D \\
<\text { SE }<-3.0 \text { D) }\end{array}$ & $\begin{array}{l}\text { Moderate Myopia } \\
\qquad \begin{array}{c}(n=55) \\
(-3.0 \mathrm{D}<\mathrm{SE} \\
<-5.0 \mathrm{D})\end{array}\end{array}$ & $\begin{array}{l}\text { High Myopia } \\
\quad(n=38) \\
(-5.0 D \leq S E)\end{array}$ & $P$ \\
\hline Age (years) & $13.52 \pm 0.64$ & $13.47 \pm 0.5$ & $13.56 \pm 0.65$ & $13.59 \pm 0.89$ & $13.54 \pm 0.59$ & 0.505 \\
\hline Gender (boy, \%) & $208(47.00)$ & $\mathrm{III(53.37)}$ & $52(25.00)$ & $29(13.94)$ & $16(7.70)$ & 0.148 \\
\hline Height $(\mathrm{cm})$ & $163.25 \pm 34.66$ & $160.7 \mid \pm 7.43$ & $169.35 \pm 61.73$ & $160.73 \pm 8.68$ & $159.92 \pm 7.58$ & 0.113 \\
\hline Weight (kg) & $53.65 \pm 12.32$ & $54.65 \pm 12.29$ & $53.16 \pm 12.72$ & $52.16 \pm 13.16$ & $51.82 \pm 9.39$ & 0.349 \\
\hline BMI $\left(\mathrm{kg} / \mathrm{m}^{2}\right)$ & $20.61 \pm 4.19$ & $21.04 \pm 3.95$ & $20.15 \pm 4.15$ & $20.29 \pm 5.42$ & $20.26 \pm 3.51$ & 0.219 \\
\hline $\mathrm{SBP}(\mathrm{mmHg})$ & $111.89 \pm 13.00$ & $111.23 \pm 13.64$ & $110.23 \pm 12.40$ & $109.91 \pm 11.45$ & $113.16 \pm 12.93$ & 0.117 \\
\hline $\mathrm{DBP}(\mathrm{mmHg})$ & $66.23 \pm 9.318$ & $66.18 \pm 9.12$ & $66.05 \pm 10.22$ & $64.63 \pm 7.60$ & $69.39 \pm 9.96$ & 0.110 \\
\hline Heart ratio & $83.74 \pm 12.28$ & $83.96 \pm 12.35$ & $81.36 \pm 11.52$ & $85.11 \pm 9.65$ & $88.92 \pm 15.84$ & 0.005 \\
\hline Sleeping time per day (min) & $514.12 \pm 63.03$ & $517.35 \pm 55.10$ & $509.21 \pm 67.46$ & $517.02 \pm 68.21$ & $508.82 \pm 80.67$ & 0.624 \\
\hline IOP $(\mathrm{mmHg})$ & $|6.79 \pm 8.0|$ & $16.73 \pm 2.74$ & $17.25 \pm 13.99$ & $16.24 \pm 3.04$ & $16.29 \pm 2.49$ & 0.837 \\
\hline Axial length (mm) & $23.82 \pm 1.72$ & $23.29 \pm 0.80$ & $23.93 \pm 1.95$ & $24.58 \pm 2.96$ & $25.31 \pm 0.70$ & $<0.001$ \\
\hline SR & $0.40 \pm 0.08$ & $0.80 \pm 0.11$ & $0.31 \pm 0.04$ & $0.21 \pm 0.1 I$ & $0.1 I \pm 0.02$ & $<0.001$ \\
\hline KI & $44.29 \pm 26.38$ & $43.88 \pm 27.33$ & $42.81 \pm 1.24$ & $50.00 \pm 51.75$ & $43.60 \pm 1.10$ & 0.382 \\
\hline $\mathrm{K} 2$ & $43.93 \pm 2.39$ & $43.83 \pm 1.50$ & $43.96 \pm 1.37$ & $43.4 \pm 5.52$ & $45.16 \pm 1.43$ & 0.004 \\
\hline $\mathrm{Km}$ & $44.11 \pm 13.29$ & $43.86 \pm 13.76$ & $43.39 \pm 1.27$ & $46.70 \pm 26.04$ & $44.38 \pm 1.23$ & 0.462 \\
\hline
\end{tabular}

Note: Data are presented as mean \pm SD.

Abbreviations: BMI, body mass index; DBP, diastolic blood pressure; IOP, intraocular pressure; SBP, systolic blood pressure; SR, Strehl ratio; KI, horizontal axes; K2, vertical axes; $\mathrm{Km}$, average corneal curvature.

Table 3 Correlation Coefficient Between Demographic, Systematic, and Ocular Parameters with SR Metric

\begin{tabular}{|c|c|c|c|c|}
\hline Variables & $\begin{array}{c}\text { Unadjusted } \\
\text { Coefficients }(\beta)\end{array}$ & $P$ & $\begin{array}{l}\text { Adjusted Coefficients } \\
\qquad(\beta)^{*}\end{array}$ & $P$ \\
\hline Age (years) & -0.002 & 0.964 & -0.011 & 0.800 \\
\hline Gender & 0.027 & 0.566 & -0.068 & 0.133 \\
\hline Height (cm) & -0.048 & 0.317 & - & - \\
\hline Weight (kg) & -0.025 & 0.595 & - & - \\
\hline BMI $\left(\mathrm{kg} / \mathrm{m}^{2}\right)$ & 0.013 & 0.787 & - & - \\
\hline $\mathrm{SBP}(\mathrm{mmHg})$ & 0.004 & 0.938 & - & - \\
\hline $\mathrm{DBP}(\mathrm{mmHg})$ & -0.024 & 0.608 & - & - \\
\hline Heart ratio & 0.014 & 0.766 & - & - \\
\hline Sleeping time per day ( $\mathrm{min}$ ) & 0.045 & 0.350 & - & - \\
\hline IOP $(\mathrm{mmHg})$ & -0.013 & 0.792 & - & - \\
\hline Axial length $(\mathrm{mm})$ & -0.400 & $<0.001$ & -0.415 & $<0.001$ \\
\hline $\mathrm{KI}$ & 0.056 & 0.243 & - & - \\
\hline $\mathrm{K} 2$ & 0.048 & 0.314 & - & - \\
\hline $\mathrm{Km}$ & 0.059 & 0.212 & - & - \\
\hline
\end{tabular}

Note: *Adjusted with age, gender and axial length.

Abbreviations: BMI, body mass index; DBP, diastolic blood pressure; IOP, intraocular pressure; SBP, systolic blood pressure; SR, Strehl ratio; KI, horizontal axes; K2, vertical axes; $\mathrm{Km}$, average corneal curvature.

with myopia would deteriorate as the $\mathrm{AL}$ increases. These findings were consistent with outcomes by Miao et al. ${ }^{5}$ Interestingly, our study is a population-based cross-sectional study, and emmetropia is used as a control group, which increases the reliability of the findings.
There are many factors that affect the visual quality, of which aberration and scattering are the main factors and they are always affected by age, refractive power of the refractive medium, pupil diameter, sharpness of the refractive medium, eye surgery and history of trauma. The 
subjects included in this study were all adolescents aged 11-18 years, with an artificial pupil diameter of $4.0 \mathrm{~mm}$, excluding the history of ocular diseases, surgery, or trauma. Therefore, the main factor affecting the visual quality of myopia in this study is the diopter of the refractive medium. In the refractive system of the human eye, the refractive power of the cornea and lens account for the main part of the total refractive power of the eyes. The visual quality depends largely on the balance and interaction between the cornea and the lens. Analyzing the reasons that the visual quality of young people's high myopia is worse than that of low and moderate myopia is mainly as follows: 1) as the refractive power increases, the anterior surface of the cornea and lens becomes steeper, and the anterior and posterior surfaces of the cornea and lens are not ideal; 2) with the increase of diopter, the shape of the eyeball changes, and the optical center of the cornea and the lens are out of axis; 3 ) with the increase of diopter, the refractive medium becomes uneven and the refractive index changes. ${ }^{13}$ These factors can cause the incident light to deviate from the ideal optical path, resulting in a decrease in visual quality. The more sleep duration (over 8 hours) maybe better for children eye health. However, there is no correlation between sleep duration and SR in the current study, and a further prospective study is needed to verify this hypothesis.

The advantage of the current study lay in the first population-based study to investigate the association between SR and degree of myopia in Chinese adolescents. However, there were some limitations in our study. Firstly, the sample size of this study was relatively small due to the strict inclusion and exclusion criteria, and only included Chinese adolescents; thus, the representativeness of current study was relatively weak. Secondly, we did not provide mydriasis for participants, which may have affected the accuracy of diopter. Thirdly, refractive error was evaluated without cycloplegia. It may cause the problem to overestimate myopia. Finally, this study only analyzed SR as optical quality outcome rather than

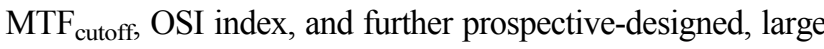
sample size and multi-ethnic studies still need to be performed.

\section{Conclusions}

Our cross-sectional study summarized the relationship and characteristics between myopia and visual quality parameter SR in adolescents in a county of northern China. Our findings show that myopia is related to SR, and SR decreases with the increasing degree of myopia. In addition, SR had an independently negative correlation with
AL. This study provides a useful basis for further confirming the influence of juvenile myopia on visual quality.

\section{Acknowledgments}

We thank the staff and participants in The Tuyou County Pediatric Eye Study for their help and support. No one received financial compensation for their contributions.

This article is supported by the National Natural Science Foundation of China (No. 81300783), China Postdoctoral Science Foundation (No. 2019TQ0358; No. 2019M661162), LiaoNing Revitalization Talents Program (No. XLYC1807082); Shenyang Young and Middle-aged Science and Technology Innovation Talent Support Program (RC190146); "Grassland Elite" Project of the Inner Mongolia Autonomous Region (No. 2020094) and Bethune Charitable Foundation (No. BJ-LM2018014J; No. BJ-LM2019008J). The funders had no role in study design, data collection and analysis, decision to publish, or preparation of the manuscript.

\section{Disclosure}

The authors report no conflicts of interest in this work.

\section{References}

1. Jones D, Luensmann D. The prevalence and impact of high myopia. Eye Contact Lens. 2012;38(3):188-196. doi:10.1097/ ICL.0b013e31824ccbc3

2. Holden B, Sankaridurg P, Smith E, Aller T, Jong M, He M. Myopia, an underrated global challenge to vision: where the current data takes us on myopia control. Eye. 2014;28(2):142-146. doi:10.1038/ eye.2013.256

3. Wu PC, Huang HM, Yu HJ, Fang PC, Chen CT. Epidemiology of myopia. Asia Pac J Ophthalmol. 2016;5(6):386-393. doi:10.1097/ APO.0000000000000236

4. Vilaseca M, Padilla A, Ondategui JC, Arjona M, Guell JL, Pujol J. Effect of laser in situ keratomileusis on vision analyzed using preoperative optical quality. $J$ Cataract Refract Surg. 2010;36 (11):1945-1953. doi:10.1016/j.jcrs.2010.05.029

5. Miao H, Tian M, He L, Zhao J, Mo X, Zhou X. Objective optical quality and intraocular scattering in myopic adults. Invest Ophthalmol Vis Sci. 2014;55(9):5582-5587. doi:10.1167/iovs.14-14362

6. Cheng X, Bradley A, Thibos LN. Predicting subjective judgment of best focus with objective image quality metrics. J Vis. 2004;4 (4):310-321. doi:10.1167/4.4.7

7. Thibos LN, Hong X, Bradley A, Applegate RA. Accuracy and precision of objective refraction from wavefront aberrations. J Vis. 2004;4 (4):329-351. doi:10.1167/4.4.9

8. Liu T, Lu G, Chen K, Kan Q, Bai J. Visual and optical quality outcomes of SMILE and FS-LASIK for myopia in the very early phase after surgery. BMC Ophthalmol. 2019;19(1):88. doi:10.1186/ s12886-019-1096-z

9. Niu L, Miao H, Tian M, Fu D, Wang X, Zhou X. One-year visual outcomes and optical quality of femtosecond laser small incision lenticule extraction and Visian Implantable Collamer Lens (ICL V4c) implantation for high myopia. Acta Ophthalmol. 2020;98(6):e662e667. doi:10.1111/aos.14344 
10. Yang Y, Zhang G, Zhang S, et al. Quantitative analysis of the macular and peripapillary capillary network with optical coherence tomography angiography in Chinese adolescents: the Tuyou County pediatric eye (TYPE) study. Int J Gen Med. 2021;14:371-379. doi:10.2147/ IJGM.S293560

11. Vilaseca M, Arjona M, Pujol J, Issolio L, Güell JL. Optical quality of foldable monofocal intraocular lenses before and after injection: comparative evaluation using a double-pass system. $J$ Cataract Refract Surg. 2009;35(8):1415-1423. doi:10.1016/j.jcrs.2009.03.022
12. Kamiya K, Umeda K, Kobashi H, Shimizu K, Kawamorita T, Uozato $\mathrm{H}$. Effect of aging on optical quality and intraocular scattering using the double-pass instrument. Curr Eye Res. 2012;37 (10):884-888. doi:10.3109/02713683.2012.688164

13. Hashemi H, Khabazkhoob M, Emamian MH, et al. Association between refractive errors and ocular biometry in Iranian adults. $J$ Ophthalmic Vis Res. 2015;10(3):214-220. doi:10.4103/2008322X.170340

\section{Publish your work in this journal}

The International Journal of General Medicine is an international, peer-reviewed open-access journal that focuses on general and internal medicine, pathogenesis, epidemiology, diagnosis, monitoring and treatment protocols. The journal is characterized by the rapid reporting of reviews, original research and clinical studies across all disease areas. The manuscript management system is completely online and includes a very quick and fair peer-review system, which is all easy to use. Visit http://www.dovepress.com/ testimonials.php to read real quotes from published authors. 